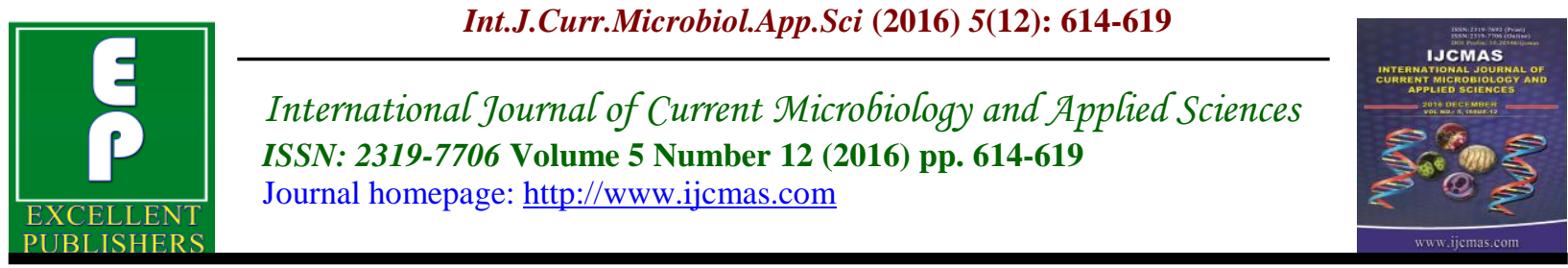

Original Research Article

http://dx.doi.org/10.20546/ijcmas.2016.512.067

\title{
Qualitative Assessment of Allergen Skin Test and In vitro Allergen Specific Immunoglobulin E (IgE) Measurement as a Method of Detection of Allergic Symptoms
}

\author{
K. Goswami ${ }^{1}$, I. Mazumdar ${ }^{2 *}$, S. Mookherjee ${ }^{3}$ and P. Pal ${ }^{4}$ \\ ${ }^{1}$ Department of Biochemistry, Lincoln University College, Kuala Lumpur, Malaysia \\ ${ }^{2}$ Department of Biochemistry, KPC Medical College, Kolkata, India \\ ${ }^{3}$ Biochemist, North City Diagnostic Centre, Kolkata, India \\ ${ }^{4}$ Department of Pharmacology, IIMSR \& B C Roy Hospital, Haldia, India \\ *Corresponding author
}

\section{Keywords \\ House Dust Mite, Asthma, \\ Skin Prick Test, Immunoglobulin E, Environmental Allergens.}

\section{Article Info}

Accepted:

20 November 2016

Available Online:

10 December 2016

\section{A B S T R A C T}

The increase in air pollutants affects both mortality and morbidity of the respiratory system. People are exposed to various indoor allergens like dust mite, pests and pet shedding, manifesting as Asthma. The diagnosis of allergy is either by allergen specific skin test (SPT) or in vitro specific allergen mediated Immunoglobulin E (IgE) measurement. Allergen specific $\operatorname{IgE}$ is indicated for evaluating allergy as a more authentic and specific diagnostic tool. But this has not yet been explored much till date. The present study assessed qualitatively the importance of both SPT and allergen specific IgE measurement for diagnosis of allergy. This study was conducted upon bronchial asthma patients. Allergy SPTs were carried out using commercially available antigens. The concentrations of allergen specific $\operatorname{IgE}$ antibodies to allergens with most positive skin test reaction were determined using the rapid, solid phase enzyme linked immunosorbent assay. Trends of reaction patterns remained similar in both. Regression analysis studies revealed positive correlation between the tests following some allergens. But corresponding correlation in all forms of allergens could not be established. Therefore characterization and standardization for the commercial allergen and specific $\mathrm{IgE}$ assay kit were proposed. In conclusion, specific IgE to aeroallergens seemed a more specific tool for detecting allergen specificity, though SPT seemed to be more sensitive.

\section{Introduction}

In the last century, the huge increase in air pollutants due to economic and industrial growth has made great alterations in the atmosphere, especially in many industrialized countries, and is fast becoming a problem for the rest of the world. The quality of air has a quantifiable impact, mostly on the respiratory system, affecting both its mortality and morbidity. Asthma is one of the most common chronic diseases which are the manifestations of allergic reactions, that has a global impact and which arises out of poor air quality. Allergy is one form of human disease which 
affects about $20 \%$ of the population. People are exposed to aeroallergens at various settings, both at home and at work. The concentration of allergens in the environment varies, depending on various factors including climate, vegetation and air quality. The outdoor allergens predominantly constitute of plant pollens and fungal spores. The indoor allergens are those represented by dust mite, pests and pets. The concentration and prevalence of the indoor allergens depend on moisture content, ventilation and the presence or absence of pets, carpets and house plants (Gravesen, 1985). Fungal spores are also reported from the indoor environment (Burge, 1989).

The diagnosis of allergy is usually established either by allergen skin test or by in vitro specific allergen mediated Immunoglobulin E (IgE) measurement (Neeper-Christensen et al., 2003). However, some patients cannot have skin tests, because of dermatographism, severe eczema and medication ( $\mathrm{Li}, 2002)$. Also, total $\operatorname{IgE}$ value is influenced by many factors such as gender, age, race and smoking habits (Arinola, 2006). Therefore measurement of allergen specific $\mathrm{IgE}$ is indicated for evaluating allergy. The use of specific IgEantibodies, against specific allergen, as a more authentic and specific diagnostic tool in allergic diseases, has not yet been explored much till date. It seems important to assess the role of specific IgE antibodies as an alternate diagnostic tool for evaluating allergic manifestations of asthma patients.

Clinically the presenting symptoms associated with allergy are sneezing, nasal discharge, coughing, wheezing, shortness of breath, angioedema, urticaria, and even anaphylaxis shock. The present study has been conducted to assess qualitatively the diagnostic importance of both specific allergen skin test and in vitro specific allergen mediated IgE measurement for diagnosis of specific types of allergy.

\section{Materials and Methods}

This study was conducted upon 382 patients who visited Chest clinic, Allergy clinic and ENT clinic from January 2016 to October 2016. Patients ranged between 15-50 years age $(29.62 \pm 11.61)$ that were diagnosed with bronchial asthma. Patients were selected based on symptoms of sneezing, watery rhinorhea, nasal obstruction, eye symptoms (in the form of redness, watering of eyes and itching), nose itching, throat or ear or any other asthma related symptoms. X ray, CT scan of paranasal sinuses, nasal endoscopy was done whenever they were needed.

Patients who were on Prednisolone in the last 3 months, or who had been taking Antihistaminics in the last 30 days were excluded from the study. Patients with history and findings of cardiovascular, renal or hepatic diseases were excluded from the study. All patients evaluated were non smokers.

A comprehensive history of the patients was taken using a structured questionnaire. This included history of cough with / without cough or sputum, shortness of breath, chest tightness, wheezing duration, frequency of acute attacks, history of allergy and previous history of asthma or other chest diseases, family or occupational history and risk of asthma, and history of previous and current drug therapy. All participants gave informed consent and the study was approved by the hospital ethical committee.

Blood sample $(3 \mathrm{ml})$ was collected from all subjects by venepuncture in non-fasting condition before the skin prick test (SPT) 
was performed. The specimen was then centrifuged for $5 \mathrm{~min}$ at $3000 \mathrm{rpm}$. Serum was collected for the assay of specific IgE measurement.. Specimens which exhibit gross hemolysis and severe lipemia were discarded. Allergy SPTs were carried out using commercially available antigens. Glycerol was used as the negative control and histamine as the positive control. The allergens applied were Dermatophagoids pteronysinnus (house dust mites or HDM), birch pollen, dog hair, cockroach, fixed feathers, cotton wool, milk and wheat grain. The diameter of wheal reaction was read after 20 mins. A wheal diameter of at least 3 $\mathrm{mm}$ after subtraction of the diameter size of the negative control was taken as a positive reaction.

The concentrations of allergen specific IgE antibodies to HDM, cockroach and dog dander (allergens with most positive skin test reaction) were determined using the rapid, solid phase enzyme linked immunosorbent assay with AllerSense specific IgE kit (Meril Diagnostics) based on the instructions of the manufacturer.

On the total $\operatorname{IgE}$ segment, low total IgE levels $(<30 \mathrm{IU} / \mathrm{ml})$ will be shown by a yellow to gray coloration, distinguishable from normal IgE levels $(60 \pm 30 \mathrm{IU} / \mathrm{ml})$ which yield moderately purple and elevated IgE levels $(<90 \mathrm{IU} / \mathrm{ml})$ which give an intense purple coloration after 30 minutes incubation with substrate or indicator.

The statistical package (SPSS, Version17.0) was used for the statistical analysis. Statistical significance was said to have been achieved with $\mathrm{p}$-value was equal to or less than 0.05 .

\section{Results and Discussion}

Data obtained on patients, who had visited the hospital clinic, according to their demographic characters (Age and Gender) is given in Table 1 .

Individuals on whom SPT was conducted and their results are depicted in Table 2.

The prevalence positivity of the skin reaction was highest with $D$. Pteronysinnus (House Dust Mite) allergen, followed by cockroach, dog fur and fungal allergen.

These people who were SPT positive for different allergens were measured for prevalence of allergen specific IgE and their results were tabulated in table 3 .

SPT was positive in $53 \%$ cases irrespective of both male and female following HDM allergy. It was observed that the concentration of specific $\operatorname{IgE}$ was positive in $45 \%$ cases irrespective of both male and female following HDM allergy, whereas $8 \%$ didn't show correlation. For the cockroach allergen, 33\% cases were measured for a positive allergic reaction whereas only about $7-8 \%$ had a positive specific $\operatorname{IgE}$ concentration. Positive SPT was observed in about $6-8 \%$ of cases of both dog dander and fungi, while around $4 \%$ of them showed positive specific IgE concentration. Trends of reactions patterns remains similar both in SPT and Specific IGE response following different allergens represented in following Fig 1 and Fig 2.

Specific IgE response is poor in comparison to SPT following other allergens like Cockroach, Dog dander and Fungal infestation, also represented in the Fig1 and Fig 2.

Regression analysis studies reveals positive correlation between SPT and Specific allergen mediated IgE response following HDM allergens, represented in the following Fig 3. 
Allergen specific $\operatorname{IgE}$ determination is widely used in the diagnosis of $\operatorname{IgE}$ mediated allergic diseases, but the relative merits of in vitro measurement of IgE antibody in comparison to in vivo skin tests are still debated (Plebani et al., 1995). In this study, aeroallergen sensitivity was found to be the reason for the commonest type of suffering that might result from climate change and air pollution. While most cases of HDM showed positive skin reaction (53\%), almost $33 \%$ of studies done on cockroach allergen reported varying prevalence, making it the second most common recognized allergen in this study. The variation in prevalence of this allergen might be a reflection of the level of cockroach infestation in the environment (Khadadah et al., 2000). Both dog dander and fungi ranked as the third common allergens in this study, with a prevalence of about $6 \%$ and $8 \%$ respectively, which is in accordance with other similar studies.

A significant proportion of the patients who have positive SPT to HDM also showed positive specific IgE to the same allergen. But corresponding correlation in case of all other forms of allergens could not be established. Only $7-8 \%$ of the people testing SPT positive for cockroach allergen (33\%) showed specific IgE levels above normal.

Table.1 Descriptive characteristics of patients who visited hospital clinic

\begin{tabular}{|l|l|l|}
\hline Characters & Male & Female \\
\hline Number of individuals (Total-285= 100\%) & $146(51.2 \%)$ & $139(48.8 \%)$ \\
\hline Age of individuals (Mean \pm SD) & $33.61 \pm 11.02$ years & $31.20 \pm 10.61$ years \\
\hline
\end{tabular}

Table.2 Number and Percentage of individuals sensitive to common allergens and their response

\begin{tabular}{|l|l|l|l|}
\hline \multirow{2}{*}{ Allergens } & \multicolumn{2}{|l|}{ No \& percentage of sensitized individuals } & $\begin{array}{l}\text { Wheal diameter } \\
(\mathbf{m m}) \pm \text { SD }\end{array}$ \\
\cline { 2 - 4 } & Male (\%) & Female (\%) & \\
\hline $\begin{array}{l}\text { D. Pteronysinnus } \\
\text { (HDM) }\end{array}$ & $77(53 \%)$ & $73(53 \%)$ & $4.05 \pm 1.16$ \\
\hline Cockroach & $48(33 \%)$ & $46(33 \%)$ & $3.36 \pm 0.61$ \\
\hline Dog dander & $11(8 \%)$ & $10(7 \%)$ & $2.99 \pm 0.10$ \\
\hline Fungal & $10(6 \%)$ & $10(\& \%)$ & $3.00 \pm 0.05$ \\
\hline
\end{tabular}

Table.3 Prevalence of allergen specific IgE in SPT positive people

\begin{tabular}{|l|c|c|}
\hline \multirow{2}{*}{ Allergens } & \multicolumn{2}{|c|}{ No \& percentage of individuals with specific IgE } \\
\cline { 2 - 3 } & Male & Female \\
\hline D. Pteronysinnus (HDM) & $66(45 \%)$ & $61(45 \%)$ \\
\hline Cockroach & $11(8 \%)$ & $10(7 \%)$ \\
\hline Dog dander & $07(5 \%)$ & $06(4 \%)$ \\
\hline Fungal & $06(4 \%)$ & $06(4 \%)$ \\
\hline
\end{tabular}


Fig.1

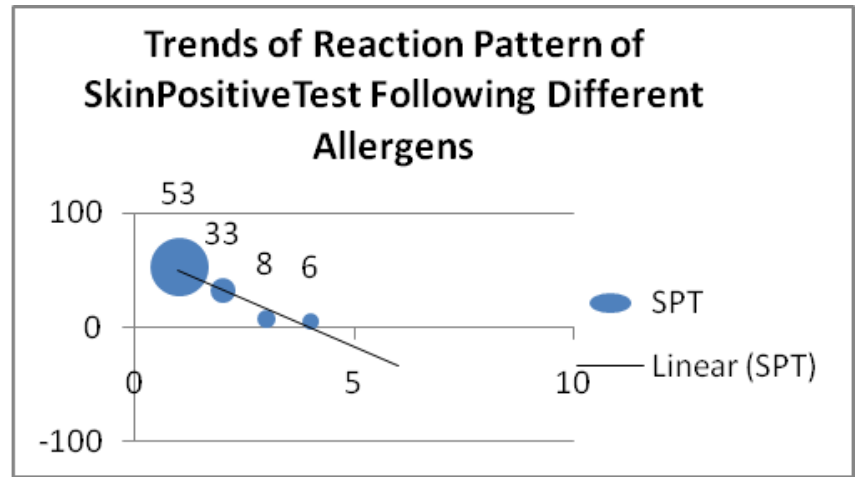

Fig.2

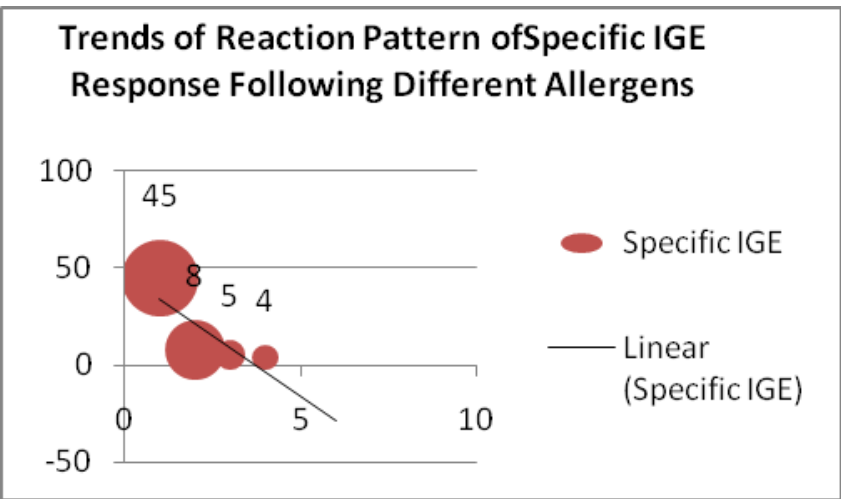

Fig.3

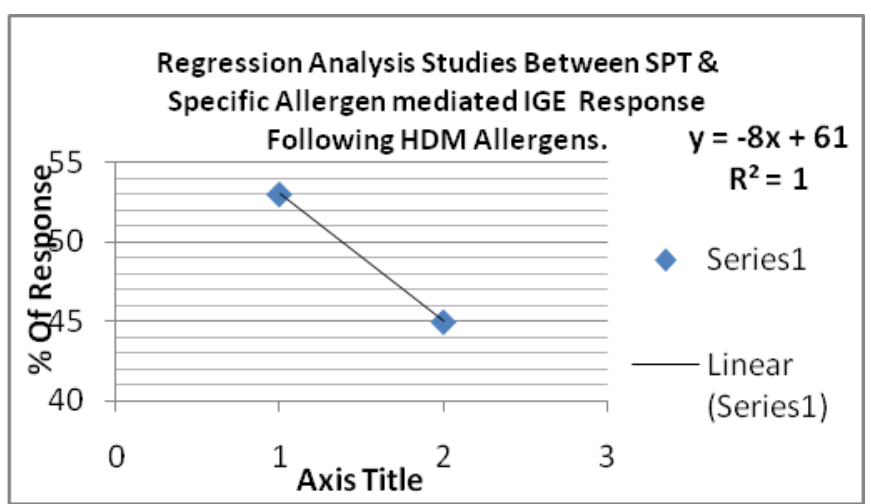

The lower sensitivity of specific IgE might be due to modification of the antigen or reagent by manufacturer. Similar findings have been reported by other studies. Therefore characterization and standardization for the commercial allergen and specific IgE assay kit are proposed.
Correlation between the size of SPT and approximate concentration of specific IgE to the four most common allergens were formed. It was seen that there is significant correlation with HDM only but not others. In other studies, a positive correlation between the wheal size of SPT and the 
specific IgE levels in the body due to cockroach bite was established. The reason for the seeming lack of association in the present study is not clear, because cockroach infestation is pretty common in our environment especially among the low socio-economic class. Perhaps, genetic, diet and environmental factors play a certain role in this observation.

Fungal antigens play an important role in the causation of respiratory allergies. Fungi disseminate their spores in the environment through air, water, insects, man and animals, making them a highly common allergen (Meczarri et al., 2002).

In conclusion, this study has revealed that specific IgE to aeroallergens is a better tool for detecting allergen specificity, though SPT seems to be the more sensitive test. Also measurement specific $\mathrm{IgE}$ is an effective method to determine sensitivity to HDM. More such studies may help in better understanding of the condition which can lead to proper diagnosis and management of such diseases.

\section{Conflict of interest}

The authors are declaring no conflict of interest in the present study.

\section{References}

Arinola, O.G. 2006. Serum Total IgE Levels in Healthy Children and Adults in
Ibadan, Nigeria. Tanzan Med. J., 23(2): 9-10.

Burge, H.A. 1989. Airborne Allergenic fungi. Immunol. Allergy Clin. North America, 9: 307-319.

Gravesen, S. 1985. Indoor airborne mould spores. Allergy, 40: 21-23.

Khadadah, M.E., Onadeko, B.O., Ezemuzie, C.I., Maroof, R., Mustafa, H.S., Sugathan, T.N. 2000. Studies on the Relationship Between allergen specific IgE Antibodies and Skin Test Reactivity in patients with Asthma in Kuwait. Med. Princ. Pract., 9: 260267.

Li, J.T. 2002. Allergy Testing. Am. Fam. Physician, 66: 621-624.

Meczarri, A. et al. 2002. Airborne Fungi in the city of Porto Alegre Rio Grande do Sul, Brazil. Ver Inst Med, Trop. S. Paulo., 44: 267-272.

Neeper-Christensen, S., Backer, V., DuBuske, L.M., Nolte, H. 2003. In vitro Diagnostic Evaluation of patients with Inhalant Allergies: Summary of Probability Outcomes Comparing Results of CLA and CAP Specific Immunoglobulin E Test System. Allergy Asthma Proc., 24(40): 253 258.

Plebani, M., Borghesan, F., Faggian, D. 1995. Clinical Efficiency of In Vitro and In Vivo Tests for Allergic Diseases. Am. Allergy Asthma Immunol., 74: 23-28.

\section{How to cite this article:}

Goswami, K., I. Mazumdar, S. Mookherjee and Pal, P. 2016. Qualitative Assessment of Allergen Skin Test and In vitro Allergen Specific Immunoglobulin E (IgE) Measurement as a Method of Detection of Allergic Symptoms. Int.J.Curr.Microbiol.App.Sci. 5(12): 614-619. doi: http://dx.doi.org/10.20546/ijcmas.2016.512.067 